\title{
Eine metaphysische Theorie der Kausalität: Michael Moores "Causation and Responsibility“
}

\author{
Jenny Nolting/Joachim Renzikowski"
}

\begin{abstract}
Rezension zu Michael S. Moore, Causation and Responsibility, Oxford (Oxford University
\end{abstract} Press) 2009, 530 S., gebunden ca. 90,- €, ISBN 978-0-19-925686-0

\section{A. Einführung}

Viele Straftatbestände verlangen, dass dem Täter der tatbestandliche Erfolg zugerechnet werden kann. So muss etwa bei $\$ 212$ StGB festgestellt werden, dass zwischen dem Verhalten des Täters und dem Tod des Opfers eine Verbindung besteht, die den Tod des Opfers als Werk des Täters ausweist. ${ }^{1}$ Ein derartiges Zurechnungsurteil setzt in praktischer Hinsicht (Willens-)Freiheit ${ }^{2}$ und in theoretischer Hinsicht Kausalität voraus. ${ }^{3}$

Üblicherweise wird die Zurechnung im Strafrecht in zwei Schritten vorgenommen. In einem ersten Schritt wird der - naturalistisch verstandene ${ }^{4}-K_{\text {Kausalzusammen- }}$ hang zwischen dem Verhalten des Täters und dem eingetretenen Erfolg ermittelt. Kausalität lässt sich zunächst definieren als ein Zusammenhang zwischen mindestens zwei Ereignissen in dem Sinne, dass das eine Ereignis als Ursache und das andere Ereignis als seine Wirkung angesehen wird. Die kausale Erklärung eines Ereignisses hat also immer die Form eines Konditionalsatzes („wenn ... dann“). ${ }^{5}$ Schließlich kann eine wahre Kausalerklärung nur tatsächlich eingetretene Ereignisse als kausal erklären. Da nach herkömmlicher Auffassung die - weite - Kausalität nur die äußerste Grenze des Haftungsrahmens beschreibt, muss die Zurechnung in einem zwei-

* Jenny Nolting ist Mitarbeiterin am Lehrstuhl für Strafrecht und Rechtsphilosophie/Rechtstheorie,, Prof. Dr. Joachim Renzikowski ist Inhaber dieses Lehrstuhls an der Martin-Luther-Universität Halle-Wittenberg. .

1 S. nur C. Roxin, Strafrecht. Allgemeiner Teil, Band 1, 4. Aufl., München 2006, $\mathbb{1 1}$ Rn. 1.

2 „Sollen impliziert Können“, s. dazu R.M. Hare, Freiheit und Vernunft, Frankfurt am Main 1983, S. 70 ff.; J. Hruschka, in: H. Ackermann/J. Albers/K. A. Bettermann (Hrsg.), Aus dem Hamburger Rechtsleben - FS Reimers, Berlin 1979, S. 459 ff.; G. H. von Wright, Norm und Handlung, Königstein 1979, S. 114 ff. - oder in der berühmten Formulierung I. Kants (Metaphysik der Sitten [1797], in: Kants gesammelte Schriften, Band 6, Berlin 1907, S. 203, 227): „Zurechnung (imputatio) in moralischer Bedeutung ist das Urtheil, wodurch jemand als Urheber (causa libera) einer Handlung, die alsdann That (factum) heißt und unter Gesetzen steht, angesehen wird." Die aktuelle, von der modernen Hirnforschung angestoßene Debatte über Determinismus und Indeterminismus kann an dieser Stelle nicht geführt werden, s. statt vieler R. Merkel, Willensfreiheit und rechtliche Schuld, Baden-Baden 2008.

3 S. nur T. Lenckner/J. Eisele in: Schönke/Schröder, StGB, 28. Aufl., München 2010, Vorbem $\mathbb{S} 13 \mathrm{ff}$. Rn. $71 \mathrm{f}$.

4 Vgl. H. Welzel, Das deutsche Strafrecht, 11. Aufl., Berlin 1969, S. 43: Kausalität als „Seinskategorie“.

5 Vgl. J. Renzikowski, Ist psychische Kausalität dem. Begriff nach möglich?, in: H.-U. Paeffgen/M. Böse/ U. Kindhäuser/S. Stübinger/T. Verrel/R. Zaczyk (Hrsg.), Strafrechtswissenschaft als Analyse und Konstruktion - FS Puppe, Berlin 2011, S. 201 (202 ff.) . 
ten Schritt normativ begrenzt werden. Der Erfolg muss sich als Realisierung einer unerlaubten Gefahr darstellen. Diesbezüglich spricht man von „objektiver Zurechnung “. ${ }^{6}$ Die Notwendigkeit einer solchen normativen Begrenzung der Zurechnung ergibt sich aus dem in der Rechtsprechung und Literatur vorherrschenden Kausalitätsbegriff der „Äquivalenztheorie“, wonach als Ursache jedes Ereignis gilt, welches nicht hinweggedacht werden kann, ohne dass der eingetretene Erfolg entfällt. ${ }^{7}$ Als Vorzug dieses Kausalitätsbegriffs wird angesehen, dass eine Differenzierung zwischen Ursachen und bloßen Bedingungen nicht möglich ist. Nicht nur der Mörder ist demzufolge ursächlich für den Tod des Opfers, sondern auch das Opfer selbst, ferner Produzent und Verkäufer der Tatwaffe, die Vorfahren dieser Personen (durch Zeugung und Geburt) usw. ${ }^{8}$ Auf diese Weise wird ein „kausaler Ozean“9 erschaffen, in dem man eigentlich nur ertrinken kann. Ungeachtet dieses Mangels, der auf der Weigerung beruht, zwischen notwendigen und hinreichenden Bedingungen zu unterscheiden, ${ }^{10}$ wird eine (weitere) Schwäche der Äquivalenztheorie darin gesehen, dass sie den Zusammenhang zwischen Ursache und Erfolg, um den es ihr geht, nicht erklärt, sondern bereits als bekannt voraussetzt. ${ }^{11}$ Verlangt man daher einen gesetzmäßigen Zusammenhang zwischen Ursache und Erfolg, ${ }^{12}$ steht man vor dem nächsten Problem, das sich allerdings für alle nomologischen Kausalitätsbegriffe in derselben Weise stellt: Es muss ein generelles Gesetz benannt werden, unter das der konkrete Geschehensablauf subsumiert werden kann. ${ }^{13}$ Als einschlägige Gesetze erscheinen zunächst die fachwissenschaftlich anerkannten Naturgesetze, doch wird in der Wissenschaftstheorie schon seit geraumer Zeit bestritten, dass sich solche deterministischen Gesetzmäßigkeiten überhaupt formulieren lassen. ${ }^{14}$ Daneben wird vorgeschlagen, auch wirtschaftliche oder soziale Gesetzmäßigkeiten für das Kausalurteil dort heranzuziehen, wo sich, wie bei menschlichen Interaktionen, keine determinis-

6 Statt vieler Roxin (Fn. 1), $\$ 11$ Rn. 2 und 11.

7 Begründer dieser Lehre sind J. Glaser, Abhandlungen aus dem österreichischen Strafrecht. Erster Band, 1858 (Nachdruck Aalen 1978), S. 298 und M. von Buri, Ueber Causalität und deren Verantwortung, Leipzig 1873, S. 1 (3), durch dessen Einfluss die Äquivalenztheorie Einzug in die Rspr. des Reichsgerichts gehalten hat, die dann vom Bundesgerichtshof fortgesetzt wurde, s. BGHSt 45, 270, $294 \mathrm{f}$.; 49 , 1, 3; aus der Literatur K. Kühl, Strafrecht. Allgemeiner Teil, 6. Aufl., München 2008, $\mathbb{S} 4$ Rn. 9 m.w.N.

8 Vgl. BGHSt 39, 195, 197 f.; Lenckner/Eisele (Fn. 3), Vorbem $\mathbb{S} 13$ ff. Rn. 76; Roxin (Fn. 1), $\mathbb{1} 11$ Rn. 6.

9 Aichele, Ex contradictione quodlibet, ZStW 2011, S. 260 (269).

10 S. Aichele (Fn. 9), ZStW 2011, S 266 ff.; weitere grundsätzliche Kritik, auf die an dieser Stelle nicht eingegangen werden muss, aus der philosophischen Diskussion bei G. Keil, Handeln und Verursachen, Frankfurt am Main 2000, S. 288 ff.

$11 \operatorname{Roxin}(F n .1), \mathbb{} 11$ Rn. $12 \mathrm{f}$.

12 K. Engisch, Die Kausalität als Merkmal der strafrechtlichen Tatbestände, Tübingen 1931, S. 20 ff.; ebenso die h.L., s. Roxin (Fn. 1), $\mathbb{S} 11$ Rn. 15; krit. R: Merkel, Über einige vernachlässigte Probleme des Kausalitätsbegriffs im Strafrecht und Ingeborg. Puppes Lehren dazu, in: FS Puppe (Fn. 5), S. 159 ff.

13 Vgl. dazu das „Hempel-Oppenheim-Schema“ s. C. G. Hempel, Studies in the Logic of Explanation, Philosophy of Science 15 (1948), $135 \mathrm{ff}$.

14 Plastisch N. Cartwright, How the Laws of Physics Lie, New York: Oxford University Press 1983; näher dazu Keil (Fn. 10), S. 151 ff. 
tischen Gesetze formulieren lassen. ${ }^{15}$ Zuletzt kommt ein nicht lediglich ästhetischer Aspekt dazu: Es leuchtet nicht ein, dass im Strafrecht und im Zivilrecht - dort ist die Adäquanztheorie verbreitet ${ }^{16}$ - unterschiedliche Kausalitätsbegriffe angewendet werden, um dieselbe Frage zu beantworten: die Ursächlichkeit eines Ereignisses für ein anderes. ${ }^{17}$

Unglücklicherweise wird die Diskussion über den „richtigen“ Kausalitätsbegriff auch in der Philosophie seit jeher kontrovers geführt, und die Jurisprudenz kann sich nicht „einfach mal so“ bei den Kollegen bedienen. ${ }^{18}$ Ungeachtet dessen muss das Recht dieses Problem lösen. Angesichts der unübersehbaren Mängel der herkömmlichen Kausalitätsbegriffe im Recht verdient jeder Versuch Interesse, einen besseren Begriff der Kausalität zu formulieren. Das gilt auch und insbesondere für Michael Moores im Jahr 2009 erschienene Monographie „Causation and Responsibility“, die in bester Tradition ähnlicher grundlegender Arbeiten (etwa Hart/Honoré, Causation in the Law, 2. Aufl. 1985) steht.

\section{B. Verantwortlichkeit als Verursachung?}

Moore beschäftigt sich, wie schon der Titel seines Buches sagt, mit der Frage nach der Ursache und damit zugleich nach dem Verursacher eines Schadens im Straf-, aber auch im zivilrechtlichen Deliktsrecht (criminal law and tort law). Scheint es sich hierbei zunächst um die einfache Zuschreibung eines hervorgerufenen Schadens (etwa ein abgebranntes Haus) zu einem mehr oder weniger schuldhaft Agierenden (z.B. einem vorsätzlichen Brandstifter) zu handeln, so kann für Moore diese Zuschreibung mit vielen Adjektiven näher bestimmt werden - „einfach“ gehört aber nur in den seltensten Fällen dazu. Dabei behandelt er viele der bereits aus der deutschen Diskussion bekannten Probleme: Wenn zwei Personen zeitgleich, aber unabhängig voneinander ein Feuer legen und diese beiden Feuer sich auf ein Haus zu bewegen und es niederbrennen (vgl. S. 86 f., 114 f.), ${ }^{19}$ wenn jemand eine Hochwasserschutzanlage außer Kraft setzt, aber das eintreffende Hochwasser derartig stark ist, dass es die

15 So etwa E. Hilgendorf, Strafprozessuale Probleme im Licht der modernen Kausallehre am Beispiel der jüngsten Produkthaftungsfälle, in: A. Eser/U. Schittenhelm/H. Schumann (Hrsg.), Festschrift für Theodor Lenckner, München 1998, S. 702 ff.; A. Hoyer, Die traditionelle Strafrechtsdogmatik vor neuen Herausforderungen: Probleme der strafrechtlichen Produkthaftung, GA 1996, S. 168 ff.; K. Rolinski, „Statistische Kausalität“ im Strafrecht, in: H.-H. Kühne (Hrsg.), Festschrift für Koichi Miyazawa, Baden-Baden 1995, S. 490 ff.; Roxin (Fn. 1), $\$ 11$ Rn. 35 ff.; zu grundsätzlichen Bedenken s. Keil (Fn. 10), S. $213 \mathrm{ff}$.

16 Vgl. BGHZ 3, 261 (266 ff.); 25, 86 (88 ff.); BGH NJW 2000, 947 (948); C. Grüneberg in: Palandt, BGB, 70. Aufl., München 2011, Vor $\$ 249$ Rn. 26 ff. m.w.N.

17 Demgegenüber plädiert Merkel, FS Puppe (Fn. 12), S. 169 gerade dafür, sich von der Illusion eines einheitlichen Kausalbegriffs zu lösen, aber das endet letztlich in Dezisionismus und Beliebigkeit.

18 Zur Vielzahl der in den Naturwissenschaften und der Philosophie vertretenen Kausalbegriffe s. etwa H. Beebee/C.. Hitchcock/P. Menzies (Hrsg.), The Oxford Handbook of Causation, Oxford: Oxford University Press 2009.

19 Zur sog. „alternativen Kausalität“s. Lenckner/Eisele (Fn. 3), Vorbem $\$ \mathbb{S} 13$ ff. Rn. 82; Roxin (Fn. 1), $\mathbb{S} 11$ Rn. 25; J. C. Joerden, Dyadische Fallsysteme im Strafrecht, Berlin 1986, S. $151 \mathrm{ff}$. 
Kapazitäten der Anlage sowieso gesprengt hätte (vgl. S. 428), wenn eine Person einen Rettungsschwimmer davon abhält, einen Ertrinkenden zu retten (vgl. S. $61 \mathrm{ff}$.), ${ }^{20}$ dann werden die Untersuchung der kausalen Relation und die Zuschreibung von Verantwortung zu zentralen Problemen für jedes richterliche Urteil.

In einem Durchgang durch die moderne Kausalitätstheorie in all ihren Facetten bemüht sich Moore, alle denkbaren rechtsrelevanten Fallgruppen auf ihre kausale Struktur hin zu untersuchen und darauf aufbauend die Fragen nach Haftbarkeit (liability), Schuld (culpability) und Verantwortung (responsibility) in sechs Hauptkapiteln zu beantworten. Das zentrale Kriterium für ihre Beantwortung heißt, folgt man dem Autor, Kausalität. Soll eine Person als Verursacher eines Schadens identifiziert werden - etwa weil die fragliche Norm voraussetzt, dass A eine andere Person "getötet" hat (vgl. S. 5 ff. zur kausalen Bedeutung von Handlungsbezeichnungen) -, so muss eine kausale Verbindung zwischen einer von ihr begangenen Handlung und einem entstandenen Schaden nachgewiesen werden. Demnach lautet Moores Hauptthese schlicht: „Causation matters“ (S. 29). Und das auf zweifache Weise: Sowohl im Kontext des Rechts, als auch bei der Klärung der Frage nach der moralischen Verantwortung eines Handelnden ist das Vorliegen einer kausalen Relation die condicio sine qua non. Dies ist zu erwähnen, da Moore eine enge Verbindung zwischen Recht und Moral voraussetzt: Gerechtigkeit ist der moralische Maßstab, an dem sich das Recht messen lassen muss (S. 4). Der Autor formuliert folgende zwar klare, ihrem Inhalt nach durchaus zweifelhafte These: Nur wer moralisch für einen Schaden verantwortlich ist, kann auch rechtlich zur Verantwortung gezogen werden. Hieraus zeigt sich die Notwendigkeit zur Offenlegung der Kausalrelation, welche zunächst als „a natural relation that holds between events or states of affairs“ (S. 5) beschrieben wird. „Because moral responsibility is tied to such a natural relation, and because the law is tied to morality, the law is also tied to this natural relation." (S. 5)

Diese natural relation ist Grundlage jeder moralischen Verantwortung und somit auch Grundlage für die Annahme von Schuld. Moralisch relevant, in der Bewertung jedoch differenziert betrachtet werden muss, ob eine Person etwas Negatives intendiert, verursacht oder das Eintreten eines Schadens nicht verhindert. Bloßes Intendieren kann nicht Grundlage von Strafe sein, erst mit dem Handeln kann man die Schuld einer Person an einem Schaden annehmen (S. 21 ff.; 34 ff.). ${ }^{21}$ Dabei stützt

20 Zum „Abbruch des rettenden Kausalverlaufs“ s. V. Haas, Kausalität und Rechtsverletzung, Berlin 2002, S. 139 ff.; J. Reinhold, Unrechtszurechnung und der Abbruch rettender Verläufe, Tübingen 2009, S. $228 \mathrm{ff}$.

21 Die dortige Diskussion erinnert an den (alten) Streit zwischen Handlungs- und Erfolgsunwert, dazu zuletzt G. Jakobs, Erfolgsunwert und Rationalität, in: W. Joecks/H. Ostendorf/T. Rönnau/T. Rotsch/ R. Schmitz (Hrsg.), Recht - Wirtschaft - Strafe - Festschrift für Erich Samson, Heidelberg 2010, S. 43 (53 f.); unabhängig davon fragt sich, ob man hier nicht - was Moore nicht tut - zwischen Strafrecht und Zivilrecht unterscheiden müsste: Ein folgenloser Versuch führt nicht zu einem ersatzfähigen Schaden, kann aber durchaus strafbar sein. 
Moore seine Überlegungen auf eine moral-sense-Perspektive: Unter den Prämissen, dass Menschen über ein korrektes moralisches Schuldempfinden verfügen und dieses Gefühl stärker ist, wenn ein Schaden verursacht wurde, als wenn er glücklicherweise ausgeblieben ist, kann geschlussfolgert werden, dass die moralische Schuld dann am schwersten ist, wenn ein Unglück durch eine Person verursacht wurde (S. 30 f.). Dieses Argument überzeugt jedoch für sich genommen nicht: Auch wenn eine absolut kontraintuitive Annahme zunächst höchst suspekt erscheint, kann nicht aus dem Übereinstimmen der Bewertung eines Sachverhalts mit der Intuition auf die Richtigkeit der Bewertung geschlossen werden. Die Irrtumsanfälligkeit und Irrationalität der menschlichen Schuldempfindung lässt sich am Phänomen der Überlebensschuld ohne weiteres zeigen. Es ist eine Sache, sich intuitiv aufgrund des eigenen „unverdienten " Überlebens im Holocaust für den Tod seiner Familienangehörigen schuldig zu fühlen; es ist eine andere Sache, jemanden getötet zu haben. Ausgangspunkt für die rechtliche sowie für die moralische Verantwortung ist das eigene Verhalten; zugerechnet werden Handlungserfolge.

Allerdings dürfen die Konsequenzen nicht losgelöst von der Handlungsabsicht, dem Grad der Verursachung, der Vermutung über das, was geschehen wäre, wenn die Handlung so nicht stattgefunden hätte und der Frage, ob eine Handlung oder eine Unterlassung vorlag, betrachtet werden. Diese vier Faktoren sind insgesamt zu berücksichtigen, bevor eine Bewertung eines Schadens im Hinblick auf Schuld und Haftung des möglichen Verursachers unter Betrachtung aller Handlungserfolge getroffen werden kann (S. 42 ff.). Die entscheidende Rolle aber kommt der kausalen Verursachung zu: "[Causation] is the big dog in this pack of four" - "absence of causation eliminates responsibility“ (S. 77). Die Bedeutung der Verursachung in Handlungskontexten ist für Moore zentral. Schuld, Haftbarkeit und Verantwortung werden zuallererst auf Basis der kausalen Wirkung von Handlungen bestimmt. Wenn der Handelnde negative Folgen nicht kausiert hat, dann fehlt die natürliche Eigenschaft der Handlung, die Grundlage für das Vorhandensein der moralischen Eigenschaft der Verantwortung ist. Der Autor nimmt eine Supervenienz-Beziehung zwischen moralischen und natürlichen Eigenschaften an, wonach der Grund für eine unterschiedliche moralische Bewertung von Handlungen, welche gleichermaßen negative Konsequenzen haben, in den unterschiedlichen Eigenschaften zu finden ist, welche diese Handlungen auszeichnen (S.42). M.a.W.: Schuld erscheint als Ableitung der natürlichen Eigenschaft der Kausalität. Diese Ausdrucksweise ist für deutsche Leser zumindest gewöhnungsbedürftig.

Nachdem die Bedeutung der Kausalität allgemein festgestellt wurde, fragt Moore im zweiten Teil des Buches nach der Natur der Kausalitätsbeziehung (S. $81 \mathrm{ff}$.). Allerdings erläutert der Autor im Folgenden nicht, wie man zunächst erwarten könnte, umfassend seinen Begriff der Kausalität, sondern entwirft, ausgehend von den von 
ihm ermittelten gesetzlichen Anforderungen, ${ }^{22}$ ein „pruned concept“", bestehend aus 13 Aspekten, welche ein Kausalitätskonzept berücksichtigen muss, will es tatsächlich anwendbar sein (S. 152 ff.). Unmittelbar einleuchtend ist zunächst Moores Hinweis, dass Korrelationen nicht mit Kausalbeziehungen verwechselt werden dürfen (S. $111 \mathrm{ff}$.). Richtig ist auch die Beschreibung der Beziehung zwischen Ursache und Wirkung als asymmetrisch und begrenzt transitiv (S. $121 \mathrm{ff} ., 134) .{ }^{23}$ Dass Kausalketten durch andere Kausalketten abgebrochen werden können (vgl. S. $123 \mathrm{ff}$., $145 \mathrm{ff}$.), wird in der deutschen Strafrechtspraxis und -wissenschaft nur für die sog. „überholende Kausalität“ anerkannt, ${ }^{24}$ obwohl der „restriktive Tatbegriff“ und die damit verbundene Unterscheidung zwischen Tätern und Teilnehmern sowie der Begriff der Handlung schlechthin es logisch ausschließen, dass die Kausalität zwischen einer Handlung und einem Ereignis durch eine weitere Handlung vermittelt wird. ${ }^{25}$ Von manchen dürfte auch seine Ansicht bestritten werden, dass es Handlungen bzw. bestimmte Eigenschaften von ihnen sind, nicht aber Unterlassungen, welche als mögliche Ursachen in Frage kommen (vgl. S. 139 ff.). Darauf ist noch zurückzukommen. Zweifel wirft jedoch die Vorstellung auf, dass eine Ursache für sich genommen weder notwendig noch hinreichend sein muss und gleichzeitig aus dem Vorliegen einer notwendigen oder hinreichenden Bedingung nicht darauf geschlossen werden, dass es sich bei dieser um eine Ursache handelt (s. $152 \mathrm{f}$.). Was bleibt, wenn der Kausalrelation sowohl notwendige als auch hinreichende Ursachen genommen werden? Mit seinem thesenartigen Katalog entwirft Moore zwar ein Kausalitätskonzept, welches auf die relevanten Fallgruppen anwendbar ist und gleichzeitig möglichen Einwänden kaum Angriffsflächen bietet. Es fragt sich aber, was es dann noch heißt, zu behaupten, eine Ursache sei kausal, wenn sie nicht „hinreichend“ für eine Wirkung ist. Anders formuliert: Wie definiert Moore seinen Begriff von Kausalität?

Vor dem Hintergrund dieser Thesen wendet sich der Autor gegen die „harm-withinthe-risk "-Analyse (HWR), die kausale Verursachung als Haftungsvoraussetzung durch das Eingehen eines (fahrlässigen, intendierten oder vorausgesehenen) Risikos ersetzt (S. 157 ff). Dieser Abschnitt ist deshalb besonders interessant, weil er sich mit Positionen auseinandersetzt, die auch von der in Deutschland vorherrschenden „Leh-

22 Schon allein aus rechtsvergleichender Perspektive lohnt sich die Lektüre seiner Analyse des der Bedingungsformel entsprechenden „but for“-tests in der englischsprachigen Rechtspraxis (S. 84 ff.).

23 Vgl. auch J. C. Joerden, Logik im Recht, 2. Aufl., Berlin 2010, S. 255 ff.

24 S. BGHSt 21, 59, 61; 39, 322, 324; Lenckner/Eisele (Fn. 3), Vorbem $\$ \mathbb{S} 13$ ff. Rn. 78 m.w.N.

25 S. auch J. C. Joerden, Strukturen des strafrechtlichen Verantwortlichkeitsbegriffs: Relationen und ihre Verkettungen, Berlin 1988, S. 35 ff.; J. Hruschka, Regreßverbot, Anstiftungsbegriff und die Konsequenzen, ZStW 110 (1998), 581 ff.; anders die h.L. vgl. C. Roxin, Bemerkungen zum Regreßverbot, in: H.-H. Jescheck/T. Vogler (Hrsg.), Festschrift für Herbert Tröndle, Berlin/New York 1989, S. 177; Lenckner/Eisele (Fn. 3), Vorbem $\mathbb{S} \mathbb{S} 13 \mathrm{ff}$. Rn. 77 m.w.N.; Moore selbst hält darüber hinaus auch Zufälle für Kandidaten zur Unterbrechung eines Kausalverlaufs. 
re von der objektiven Zurechnung " vertreten werden. ${ }^{26}$ Ausgehend von zwei grundlegenden Annahmen - ein Angeklagter hat dann fahrlässig gehandelt, wenn er abschätzen konnte, welches Risiko er durch die Handlung auf sich nimmt und wenn der entstandene Schaden ein absehbares Risiko ebendieser Handlung ist (S. 157) unterzieht Moore die HWR einer umfassenden Kritik um zu zeigen, dass sie ihren Anspruch, die Verursachungsrelation bei Fahrlässigkeitsdelikten durch die Annahme von risikobegründeter Schuld zu ersetzen, nicht erfüllen kann. Moore stellt fest, dass die Verfechter der HWR eine Handlung dann als fahrlässig betrachten, wenn jemand durch dieses Tun einem unverantwortbaren Risiko ausgesetzt wird - nicht wenn er tatsächlich Schaden erleidet. Tritt ein Schaden ein, fragt die HWR danach, ob dieser ein voraussehbares Risiko der Handlung war. Die Relation, die in Bezug auf die fahrlässige Handlung zwischen Kläger und Angeklagtem besteht, ist dann keine Kausalrelation, sondern eine risikobasierte Relation. Allerdings trägt diese Sichtweise dem Umstand nicht Rechnung, dass es die pflichtverletzende Handlung und die rechtsverletzenden Folgen sind, welche man moralisch verpflichtet ist zu unterlassen. Ein Risiko einzugehen ist nicht per se verboten - ein Führerschein erlaubt es ein Auto zu steuern, auch wenn damit das permanente Risiko ein Kind zu töten besteht. „The result is that the law cannot make risking into a violation of some victim's rights. Our legal rights are not rights against being hit by some risk; they are rights against actually being caused harm.” (S. 172) Darin klingt der Vorwurf einer Vermischung von Gewährleistungsfunktion des subjektiven Rechts und defensivem Rechtsschutz einerseits und Reichweite der Verhaltensnorm andererseits an. ${ }^{27}$

Eine weitere Schwierigkeit der HWR besteht laut ihrer Interpretation durch Moore darin, dass jede Folge innerhalb des möglichen Risikos einer Handlung lag - es kann also nicht zwischen voraussehbaren und nicht voraussehbaren Handlungsfolgen unterschieden werden, da jeweils nur die Handlungsfolgen eintreten können, für deren Eintreten ein Risiko bestand (S. 185 ff.). Von der Aktualisierung eines Schadens kann immer auf dessen Möglichkeit geschlossen werden. Somit kann kein Verhalten als erlaubt erscheinen, da jede Handlung eine unendliche Zahl möglicher Risiken birgt: Jeder mögliche Schaden ist „within the risk“ (S. 194). Dieser Kritikpunkt trifft auch die „Lehre von der objektiven Zurechnung “: Da sie keine - oder lediglich zirkuläre - Kriterien für die Unerlaubtheit des Risikos angeben kann, leistet sie nicht, was sie vorgibt: eine normative Beschränkung der uferlosen Kausalität. ${ }^{28}$

26 Hier sind vor allem diejenigen Autoren zu nennen, die die Kausalität durch die Risikoerhöhung ersetzen wollen, s. etwa A. Hoyer, FS Rudolphi, 2004, S. 95, 102 f.; Pérez-Barberá ZStW 114 (2002), 600 (628 ff.); man kann allerdings mit Ferrante, New Criminal Law Review 14 (2011), 162 (168 ff.) bestreiten, ob man die HWR in diesem Sinne rekonstruieren kann.

27 S. auch Haas (Fn. 20), S. $58 \mathrm{ff.}$

28 Näher dazu Haas (Fn. 20), S. 293 ff.; Aichele (Fn. 9), ZStW 2011, S. 271 f. 
Neben diesen konzeptuellen Einwänden setzt sich Moore auch mit den normativen Schwierigkeiten der HWR auseinander (S. 198 ff.). Die Vertreter dieser Theorie gehen davon aus, dass jemand nur dann die Schuld an einem entstandenen Schäden trägt, wenn dessen Eintreten von vernünftigen, verantwortungsvollen Personen als wahrscheinlich hätte erachtet werden müssen. Wenn also die Handlung des Beschuldigten zur Aktualisierung eines äußerst unwahrscheinlichen Risikos führe, dann könne von ihm kein Ausgleich für den entstandenen Schaden gefordert werden. Betrachtet man jedoch den Umstand, dass jeder entstandene Schaden zuvor ein mögliches Risiko darstellte, kann kaum sinnvoll zwischen wahrscheinlichen und unwahrscheinlichen Risiken unterschieden werden. Begründet werden müsste auch, warum ein jemand für einen Schaden, den er verursacht hat, nicht verantwortlich gemacht werden sollte, nur weil sein Eintreten höchst unwahrscheinlich war (S. 199). Zusammenfassend stellt Moore fest: „As applied in negligence cases, the analysis is both conceptually incoherent and normatively undesirable. As applied to all cases, intent and foresight as well as negligence, HWR gives an inaccurate description of the decided cases.” (S. 225). Damit verwirft er die „harm-within-the-risk“-Analyse als Möglichkeit der Begründung von (Straf-)Haftung auf der Grundlage des Vorliegens von Schuld und hält an der eingangs postulierten These „Causation matters.“ fest.

Allerdings muss gefragt werden, ob die moore'sche Fundamentalkritik die HWR wirklich im Kern trifft. Immerhin scheint Moore außer Acht zu lassen, dass sie sich nicht variabel auf ausnahmslos alle Risiken bezieht, welche permanent der Möglichkeit nach bestehen, sondern vielmehr nur auf solche Gefahren, welche durch eine unerlaubte Handlung geschaffen wurden. Nur die so als unerlaubte Gefahrschaffung ausgezeichneten Handlungen spielen im Zurechnungsurteil eine Rolle. Somit ist keineswegs jeder Schaden „within the risk “ und damit Gegenstand möglicher Zurechnungsnormen, sondern nur der, welcher aus einer unerlaubten Handlung erwächst, die die gebotene Sorgfalt nicht beachtet. Gleichwohl muss kritisiert werden, dass die Lehre aus einer ex post Perspektive danach fragt, ob das Risiko ein mögliches Risiko der Handlung war - was in jedem Fall bejaht werden kann, da von der Wirklichkeit jederzeit auf die Möglichkeit geschlossen werden kann. Völlig ausgeblendet werden jedoch die Perspektive des Handelnden sowie seine Kenntnisse und Annahmen und stattdessen unter idealisierten Umständen gefragt, ob ein Risiko als solches hätte erkannt werden müssen. Keine Beachtung findet dabei die Person des Handelnden, die jedoch Subjekt eines singulären Zurechnungsurteils sein soll. ${ }^{29}$

Der vierte Teil greift nochmals die bereits angesprochene Problematik der „Unterbechung des Kausalverlaufs“ auf (S. 229 ff.) und diskutiert Probleme wie die eines

29 Vgl. hierzu auch den Streit darüber, ob das unerlaubte Risiko nach generellen oder nach individuellen Maßstäben zu bestimmen ist, s. etwa Roxin (Fn. 1), $\$ 24$ Rn. 53 ff. Kindhäuser, Zum sog. „unerlaubten" Risikoin, in: R. Bloy/M. Böse/T. Hillenkamp/ C. Momsen/P. Rackow (Hrsg.), Gerechte Strafe und legitimes Strafrecht - Festschrift Maiwald, Berlin 2010, S. 397 (401ff.). 
Regressverbots, welches hierzulande von der ganz h.L. auf der Basis der Äquivalenztheorie abgelehnt wird. Dabei kann man die Ausführungen über „voluntary human actions“ (S. $241 \mathrm{ff}$.) als englischsprachige Variante des „Verantwortungsprinzips“ ${ }^{30}$ lesen. Moore zufolge soll es nicht darauf ankommen, ob die Zweithandlung aus der Perspektive des Ersthandelnden vorhergesehen wurde oder zumindest erkennbar gewesen ist (S. 249 ff.). ${ }^{31}$ Allerdings vermisst Moore eine metaphysischen Grundlegung des Kausalitätsbegriffs (S. 254 ff.), gibt sich dabei aber auch nicht mit dem Hinweis zufrieden, dass eine Handlung per se frei und damit unverursacht ist (S. 268 ff., ferner S. 288 ff.). Seine Position bleibt an dieser Stelle etwas unklar: Was versteht er unter „sufficiently big causes“ (S. 274 ff.)? Für die Begründung der zivilrechtlichen Haftung und der Strafbarkeit von Teilnehmern hält Moore jedenfalls den Rückgriff auf Kausalität für irreführend und entbehrlich (S. 280 ff.). ${ }^{32}$

Standen bis dahin vor allem die Fragen nach responsibility und culpability im Vordergrund, so legt Moore im fünften Teil den Fokus auf die Verursachungsrelation (S. 327 ff.). Als Relata einer Kausalrelation kommen zunächst verschiedene Gegenstände in Betracht. So wird äußerst kontrovers diskutiert, was als mögliche Ursache innerhalb einer kausalen Relation bezeichnet werden kann - auch der Rückgriff auf die Rechtsprechung vermag hier keine eindeutige Bestimmung zu liefern. Unter einer Vielzahl potentieller Ursachen identifiziert Moore events und facts als mögliche Bestandteile einer Kausalrelation (S. 329). Ereignisse werden dabei verstanden als raum-zeitlich existierende Einzeldinge mit verschiedenen notwendigen wie kontingenten Eigenschaften, welche sich in einem bestimmten Zeitraum in einem bestimmten Zustand befinden und im Prozess des Zustandswechsels eine Wirkung kausieren (S. 334 ff.). Fakten treten allein in Form wahrer Propositionen über die Eigenschaften einer aktual existenten Entität auf. Sie beziehen sich auf die Welt, sie referieren auf Dinge, sind aber nicht ihrerseits Teil der Welt (S. $341 \mathrm{ff}$.). Eine Kausalrelation setzt einen Zustandswechsel voraus, also ein Kausieren einer Wirkung durch eine Ursache. Sätze jedoch kausieren schlechterdings nichts; sie beschreiben und referieren, aber sie sind nicht ihrerseits Bestandteile eines kausalen Prozesses (S. 347). Mögliche Relata einer Kausalrelation sind vielmehr die Wahrmacher einer wahren Proposition, also die Dinge, die ihr korrespondieren und über welche mittels eines Urteils etwas ausgesagt wird.

Somit können Ereignisse als Bestandteile der Kausalrelation identifiziert werden. Die kausale Relation besteht allerdings nicht zwischen Ereignissen per se, sondern zwi-

30 S. Joerden (Fn. 25), S. 30 f., 35, 88 ff.; Renzikowski, Restriktiver Täterbegriff und fahrlässige Beteiligung, 1997, S. 73 ff.; bei der Abgrenzung von Täterschaft und Teilnahme abl. Roxin, Täterschaft und Tatherrschaft, 8. Aufl. 2006, S. 683 f.; Schünemann in: Leipziger Kommentar zum StGB, Bd. 1, 12. Aufl. 2007, \$25 Rn. 13.

31 Anders etwa Roxin (Fn. 1), $\mathbb{} 24$ Rn. $28 \mathrm{f}$.

32 Ähnlich etwa Haas, Die Theorie der Tatherrschaft und ihre Grundlagen, 2008, S. $130 \mathrm{ff}$. 
schen den Eigenschaften, die bestimmten Ereignissen zukommen. Jede singuläre Kausalrelation ist eine Aktualisierung eines allgemeinen Kausalgesetzes. Um also ein singuläres Ereignis unter ein allgemeines Gesetz fassen zu können, müssen die Ereignisse identisch sein. Metaphysische Entitäten sind jedoch nicht im Sinne der Ununterscheidbarkeit identisch, vielmehr sind es bestimmte Eigenschaften, welche die eigentliche Ursache innerhalb einer Kausalrelation darstellen (S. 361 ff.). Wenn also bestimmte Eigenschaften einer Handlung identifiziert werden können, welche als Ursache eine bestimmte Wirkung - im rechtlich relevanten Sinne einen Schaden hervorrufen, dann kann eine Person für den entstandenen Schaden verantwortlich gemacht werden. Moore identifiziert als Basis der Kausalrelation also metaphysische Entitäten und entwickelt darauf aufbauend einen metaphysischen Kausalbegriff. Die Eigenschaften der Dinge, welche sich in bestimmten Zuständen befinden, sind Ursachen. Ein Kausalurteil kann dann gefällt werden, wenn bestimmte metaphysische Eigenschaften als Ursachen bestimmte metaphysische Wirkungen, welche sich ihrerseits im Auftreten von Ereignissen mit bestimmten Eigenschaften zeigen, nach sich ziehen (S. 368).

Der abschließende sechste Teil beschäftigt sich mit der Metaphysik der Kausalrelation. Bevor er seinen eigenen singulären Kausalbegriff näher erläutert, setzt sich Moore mit den beiden wohl am meisten verbreiteten Konzepten von Kausalität auseinander, dem kontrafaktischen und dem nomologischen Begriff der Kausalität. Angesichts der nahezu unangefochtenen Herrschaft der Äquivalenztheorie im deutschen Strafrecht, die nach dem „Was wäre gewesen, wenn? “ fragt, verdienen die ersten drei Kapitel zu den logischen Voraussetzungen und Problemen eines kontrafaktischen Kausalitätsbegriffs (S. $371 \mathrm{ff}$.) eine eingehende Lektüre. Ein kniffliges Problem ist dabei die Frage, unter welchen Bedingungen ein Satz wie „Wenn x nicht gewesen wäre, wäre auch y nicht eingetreten " wahrheitsfähig ist. ${ }^{33}$ Das zweite Problem besteht darin, dass nicht alle kontrafaktischen Konditionalsätze Kausalrelationen bezeichnen. ${ }^{34}$ Schließlich gerät der kontrafaktische Kausalitätsbegriff bei der „Mehrfachkausalität" in Schwierigkeiten. ${ }^{35}$

Bis zu diesem Punkt lässt Moore keinen Zweifel an der Relevanz der Kausalität für die Feststellung von Schuld aufkommen. Gleichwohl gibt es Fälle, in denen dieses Konzept nicht angewendet werden kann, da schlicht die kausalen Relata fehlen: Un-

33 Engischs Antwort, die Lehre vom gesetzmäßigen Zusammenhang zwischen Ursache und Erfolg (s. Fn. 12), ist dabei nicht die einzige Schwierigkeit (vgl. dazu S. 374 ff.).

34 S. auch Merkel, FS Puppe (Fn. 12), S. 156 ff.

35 Dieses Problem ist auch aus der Diskussion der „Äquivalenztheorie“ bekannt und lässt sich keineswegs durch eine „Modifikation“ (vgl. BGHSt 39, 195 (198)) aus der Welt schaffen - denn tatsächlich wird die Äquivalenztheorie damit aufgegeben. Eine konsequente Antwort läuft darauf hinaus, in solchen Konstellationen Kausalität abzulehnen, weil sich die einzelne Ursache nicht identifizieren lässt, so etwa F. Toepel, Kausalität und Pflichtwidrigkeitszusammenhang beim fahrlässigen Erfolgsdelikt, Berlin 1992, S. 72 ff. - für Moore (S. 430 ff.) eine kontraintuitive Lösung 
terlassungen (S. 435 ff.). Diese zeichnen sich dadurch aus, dass kein singuläres Ereignis stattfand, welchem die Eigenschaft zugeschrieben werden kann, Ursache zu sein. „[N]egative events, negative states of affairs, and negative properties do not exist” (S. 444) - m.a.W.: „ex nihil nihilo fit“. ${ }^{36}$ So ist ein „Schießen mittels einer Pistole“ metaphysisch zu unterscheiden von einem „Nicht-Schießen“. Der Einzelhandlung „Schießen mittels einer Pistole“ können verschiedene Eigenschaften zugeordnet werden, sie kann andere Ereignisse nach sich ziehen, sie kann Ursache für einen Schaden sein. Wenn man auf den Handlungstyp „Nicht-Schießen“ referieren möchte, dann allein über negative Propositionen - allerdings wurde schon gezeigt, dass Fakten, d.h. Aussagen über Ereignisse keine geeigneten Kausalrelata sind. Es kann kaum sinnvoll behauptet werden, dass die Proposition „X hat $\mathrm{Y}$ erschossen“ eine kausale Wirkung nach sich zieht. Ebenso wenig sind Aussagen über Nicht-Ereignisse mögliche Relata. Dies ist in der deutschen Debatte allerdings nicht unumstritten. So argumentiert etwa Puppe (in diesem Heft), dass es „keinen Grund [gibt], die Unterlassung eines bestimmten gebotenen Verhaltens nicht als Ursache zu behandeln. " Zumeist wird dieser Streit aber als fruchtlos angesehen.Für Moore ist allerdings eine kausale Verbindung zwischen einer Handlung und einem daraus folgenden Schaden nicht die einzige Möglichkeit, um ein Urteil über mögliche Verantwortung und Haftbarkeit zu fällen. Vielmehr ist zu prüfen, ob eine Kausalkette, die zu einem Schaden führte, hätte unterbrochen werden müssen, ob also jemand einer Unterlassung für schuldig befunden werden muss, da er in einer bestimmten Situation die Pflicht sowie das Vermögen hatte, einen vorhersehbaren Schaden abzuwenden (S. 439 ff.). Die Annahme einer kontrafaktischen Abhängigkeit, obwohl weder notwendig noch hinreichend für Kausalität (S. 391 ff.), soll in Fällen von Unterlassung die Feststellung von Verantwortung ermöglichen. Es bleibt die Frage, auf welche Weise bestimmt werden soll, was entgegen der Fakten hätte geschehen müssen, um den schädigenden Kausalverlauf zu unterbrechen.

An keiner Stelle jedoch klärt Moore über das Konzept der Zurechnung auf, welches seinem Handlungs- und Unterlassungsbegriff zugrunde liegt. Moore kritisiert sowohl die Lehre der objektiven Zurechnung als auch die Annahme kontrafaktischer Abhängigkeiten als Wege zur Feststellung von Kausalität und Verantwortung. Gleichwohl gelingt es ihm nicht aufzuzeigen, in welchen Fällen eine Handlung als Tat zugerechnet werden kann und muss. Moore kann schlicht mit dem Begriff der Zurechnung in der kantischen Tradition nichts anfangen. Der Grund für diese konzeptuelle Blindheit, die ihn nicht über die kausale Relation hinausblicken lässt, liegt in seiner Interpretation ebendieser Relation, wie im Folgenden gezeigt werden soll.

36 Pointiert Joerden, Strukturen (Fn. 25), S. 48 f.; dagegen etwa D. Birnbacher, Tun und Unterlassen, Stuttgart 1995, S. $75 \mathrm{ff}$. 
Moore fragt abschließend nach der Verbindung der kausalen Relata. Es lässt sich feststellen, dass beim Vorliegen einer singulären kausalen Beziehung auf ein dahinter liegendes allgemeines Gesetz geschlossen werden kann. Allerdings stehen sich zwei Theorielager bei der Frage gegenüber, ob es sich bei dem Verhältnis zwischen Kausalgesetz und singulärer Kausalbeziehung um ein induktives oder ein deduktives handelt. ${ }^{37}$ Moore verweist auf die Schwierigkeit, nichtkausale von kausalen Regularitäten zu unterscheiden und meint, es sei vor allem in den Fällen der Mehrfachkausalität nicht möglich, eine hinreichende (Mindest-)Bedingung zu formulieren (S. 471 ff.). ${ }^{38}$ Daher schlägt er sich klar auf die Seite der Singularitäts-Theoretiker, denen zufolge eine singuläre Kausalbeziehung zunächst für sich genommen besteht und nicht auf generelle Gesetze zurückgeführt werden kann, mögen sie auch durchaus bestehen. Er selbst sieht sich als Vertreter eines moderate singularism (S. 496), ergänzt durch einen metaphysical primitivism (S. 505). Grundlegend für Kausalität ist laut Moore also die singuläre Beziehung zwischen metaphysischen Entitäten, welche den Ausgangspunkt für die induktive Ableitung eines allgemeinen Kausalgesetzes bildet. Kausalität wird als metaphysische Relation identifiziert, auch hier wie so oft in seinem Werk mit Verweis auf die Übereinstimmung dieser Auffassung mit der Intuition als Maßstab für die Überzeugungskraft von Argumenten.

Hierin liegt, bei aller Anerkennung seiner stupenden und scharfsinnigen Darstellung der gängigen Konzepte von Kausalität und Unterlassung, von Schuld und Verantwortung, die zentrale Schwäche seines eigenen Konzepts. Kausalität ist keine metaphysische Relation. Metaphysische Entitäten, wie sie der Autor versteht, zeichnen sich dadurch aus, dass sie in Raum und Zeit vorkommen, dass sie dinglich verfasst und somit wahrnehmbar sind. Kausalität jedoch kann man nicht betrachten oder berühren; kurz: Kausalität kann man nicht wahrnehmen. ${ }^{39}$ Es handelt sich dabei vielmehr um eine Möglichkeit, die logische Beziehung zwischen zwei Ereignissen zu benennen und begrifflich zu beschreiben. Ursachen sind als Ursachen aber keine metaphysischen Entitäten. Wir sehen zwei Ereignisse in raum-zeitlicher Nähe, schreiben einem Ereignis zu, Ursache einer Wirkung zu sein und nehmen dann eine kausale Verbindung an. Das Ereignis ist zwar empirisches Fundament der Kausalität, die kausale Verbindung selbst ist aber als Relation nicht unabhängig von unserem Den-

37 In der deutschen Strafrechtswissenschaft hat die Theorie von der hinreichenden Mindestbedingung eine gewisse Anhängerschaft gefunden, s. etwa I. Puppe, Der Erfolg und seine kausale Erklärung im Strafrecht, ZStW 1980, S. 874 ff.; I. Puppe, in: Nomos Kommentar zum StGB, 3. Aufl., Baden-Baden 2010, Vor $\$ 13$ Rn. 102 f.; ferner U. Kindhäuser, Kausalanalyse und Handlungszuschreibung, GA 1982, S. 485 ff.; zur Kritik vgl. M. Binns, INUS-Bedingung und strafrechtlicher Kausalbegriff, BadenBaden 2001, S. $73 \mathrm{ff}$.

38 Mit einigen Einwänden setzt sich F. Toepel, Hinreichende Mindestbedingung, in: FS Puppe (Fn. 5), S. $289 \mathrm{ff}$. auseinander.

39 Dazu hat schon D. Hume, An Enquiry Concerning Human Understanding (1748), Nachdruck der Ausgabe von 1777, 3. Aufl. Oxford 1975, S. 73 ff. alles Notwendige gesagt. Moore freilich ist nicht überzeugt (s. S. 263 ff.). 
ken und Erkennen. Wird weiterhin die Beziehung zwischen Ursache und Wirkung in erster Linie als singulär verstanden, sieht sich Moore dem sog. „Induktionsproblem“ gegenüber, nämlich der Unmöglichkeit, aus singulären Ereignissen wahre allgemeine Gesetze abzuleiten.

Betrachtet man Kausalität dagegen als logisch-begriffliche Relation, wonach der Verbindung zwischen Ursache und Wirkung allgemeine Gesetze zugrunde liegen, dann stellt sich zum einen das Induktionsproblem nicht. Zum anderen wird offensichtlich, dass eine kausale Beziehung zwischen einem Handelnden und der Wirkung seiner Handlung keinesfalls ausreichen kann, um von Schuld zu sprechen, ja dass die Inanspruchnahme des Schuldbegriffs jede kausale Relation transzendiert. Ein Zurechnungsurteil kann nicht mit einem Kausalurteil gleichgesetzt werden, da ein evidenter Unterschied zwischen beiden Urteilen besteht: Mittels eines Ursache-Wirkungszusammenhanges wird auf ein allgemeines Gesetz referiert, ein Zurechnungsurteil ist dagegen stets ein singuläres Urteil über einen Normverstoß dieser Person. ${ }^{40}$ Dieses Problem wird in der philosophischen Literatur unter dem Begriff der „Applikationsaporie“ erörtert. ${ }^{41}$ Führt man sich die kategoriale Andersartigkeit dieser beiden Urteilstypen und ihre völlig unterschiedliche Bedeutung für die Zurechnung vor Augen, muss gefragt werden, ob die Ausgangsthese des Autors „Causation matters“ nicht besser durch „Imputation matters“ ersetzt werden sollte.

Aber auch wenn, was hier angeklungen ist, Moores eigenes metaphysisches Konzept von Kausalität nicht überzeugt, so lohnt sich die Lektüre seines Buches gleichwohl: Sie bietet einen kritischen state of art-Überblick über die zeitgenössische Diskussion der Kausalität im englischsprachigen Raum, und viele der in diesem Buch scharfsinnig ausgebreiteten Überlegungen sind für die deutsche Diskussion wichtig, weil hier über die gleichen Fragestellungen gestritten wird. Moores Analyse lässt vieles in einem anderen, helleren Licht erscheinen.

40 Dazu erneut Aichele, Persona physica und persona moralis: Die Zurechnungsfähigkeit juristischer Personen nach Kant, JRE 16 (2008), S. 1 ff.

41 S. dazu die knappen Hinweise bei Aichele (Fn. 9), ZStW 2011, $281 \mathrm{ff.}$ 